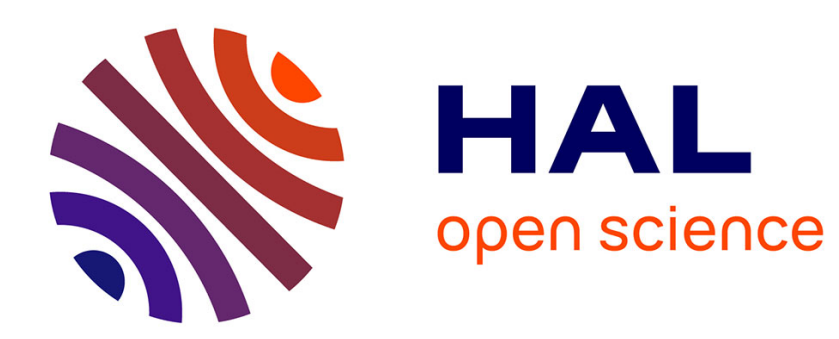

\title{
Comparative risk aversion of different preferences
}

Richard Ruble

\section{- To cite this version:}

| Richard Ruble. Comparative risk aversion of different preferences. 2011. halshs-00585615

\section{HAL Id: halshs-00585615 \\ https://shs.hal.science/halshs-00585615}

Submitted on 13 Apr 2011

HAL is a multi-disciplinary open access archive for the deposit and dissemination of scientific research documents, whether they are published or not. The documents may come from teaching and research institutions in France or abroad, or from public or private research centers.
L'archive ouverte pluridisciplinaire HAL, est destinée au dépôt et à la diffusion de documents scientifiques de niveau recherche, publiés ou non, émanant des établissements d'enseignement et de recherche français ou étrangers, des laboratoires publics ou privés. 


\section{GATE Groupe d'Analyse et de Théorie Économique Lyon-St Étienne}

93, chemin des Mouilles 69130 Ecully - France

Tel. +33 (0)4 72866060

Fax $+33(0) 472866090$

6, rue Basse des Rives 42023 Saint-Etienne cedex 02 - France

Tel. +33 (0)4 77421960

Fax. $+33(0) 477421950$

Messagerie électronique / Email : gate@gate.cnrs.fr

Téléchargement / Download : http://www.gate.cnrs.fr - Publications / Working Papers 


\title{
Comparative Risk Aversion of Different Preferences
}

\author{
Richard Ruble*
}

April 7, 2011

\begin{abstract}
An article by Kihlstrom and Mirman about comparative risk aversion with many goods is critiqued. If "more risk averse" is interpreted as signifying that an individual is less willing to accept a median-preserving spread, then risk aversion cannot be compared across individuals with different preferences. If it is interpreted as signifying that an individual has a greater directional risk premium, then risk aversion may be compared across individuals with different preferences, in particular in partial equilibrium analysis.

Keywords: Risk aversion, Risk premium

J.E.L. Classification: D81, G11
\end{abstract}

*Université de Lyon, Lyon, F-69003, France; EM Lyon, Ecully, F-69134; CNRS, GATE Lyon St Etienne, Ecully, F-69130, France. Email: ruble@em-lyon.com. I am grateful to Arnold Chassagnon and François Le Grand for helpful comments. 


\section{Introduction}

In the wake of the seminal study of risk aversion by Arrow [1] and Pratt [5], it must have seemed natural to ask whether a similar analysis might be carried out in a setting with many goods. This project was quickly curtailed by an example in Kihlstrom and Mirman [3] that purports to show that comparative risk aversion is not well-defined when individuals have different preferences. The concept of comparative risk aversion in several dimensions having been so restricted, the subject finds some mention in textbooks thereafter (notably in Laffont [4]) and even disappears from view in others, while continuing to occasionally draw the interest of researchers (most recently, Bommier, Chassagnon, and Le Grand [2]).

In this paper, section 2 argues that Kihlstrom and Mirman's example rests upon their particular choice of lottery, so that if one restricts lotteries in the same way these authors do in their subsequent analysis, it does not follow that interpersonal comparisons of risk aversion are impossible when there are many goods. Section 3 draws on a framework taken from Bommier et. al., and provides a foundation for Kihlstrom and Mirman's restriction, if risk aversion is interpreted as aversion to median-preserving spreads. Section 4 shows that if risk aversion is measured by directional risk premia, then it may be compared across some individuals with different preferences. For example, with quasilinear preferences, if a single-crossing condition holds then such comparisons are possible and reflect one-dimensional Arrow-Pratt risk aversion.

Thus, with the hindsight of nearly forty years, two central tenets of Kihlstrom and Mirman are reaffirmed, albeit independently. Either risk-aversion is to be understood with respect to a fine ordering on risky alternatives, in which case comparative risk aversion is indeed meaningful only with identical preferences, or it is understood with respect to certainty equivalents, in which case the comparison of risk aversion between different preferences is possible on the basis of a measure of risk aversion, the risk premium, that Kihlstrom and Mirman themselves had extended to the case of several dimensions.

\section{A Particular Example}

Figure 1 is as in Kihlstrom and Mirman [3]. Their argument runs as follows. Consider the following pair of lotteries: $X_{1}$ for sure, and $X_{1}$ or $X_{2}$ with equal probability (denoted $\tilde{X}$ ).

The preferences in the figure are such that $u_{1}\left(X_{1}\right)>E u_{1}(\tilde{X})$ whereas $u_{2}\left(X_{1}\right)<E u_{2}(\tilde{X})$. Therefore, consumer 2 cannot be said to at least as risk-averse as consumer 1 since the latter acts more risk-aversely, presumably by not choosing the lottery that consumer 2 is willing to choose over the sure thing. As this reasoning holds for any cardinal representation of the preferences, the differing choices of the two individuals with respect to risk are due entirely to (ordinal) preferences rather than to differing risk attitude. The authors then assert: 


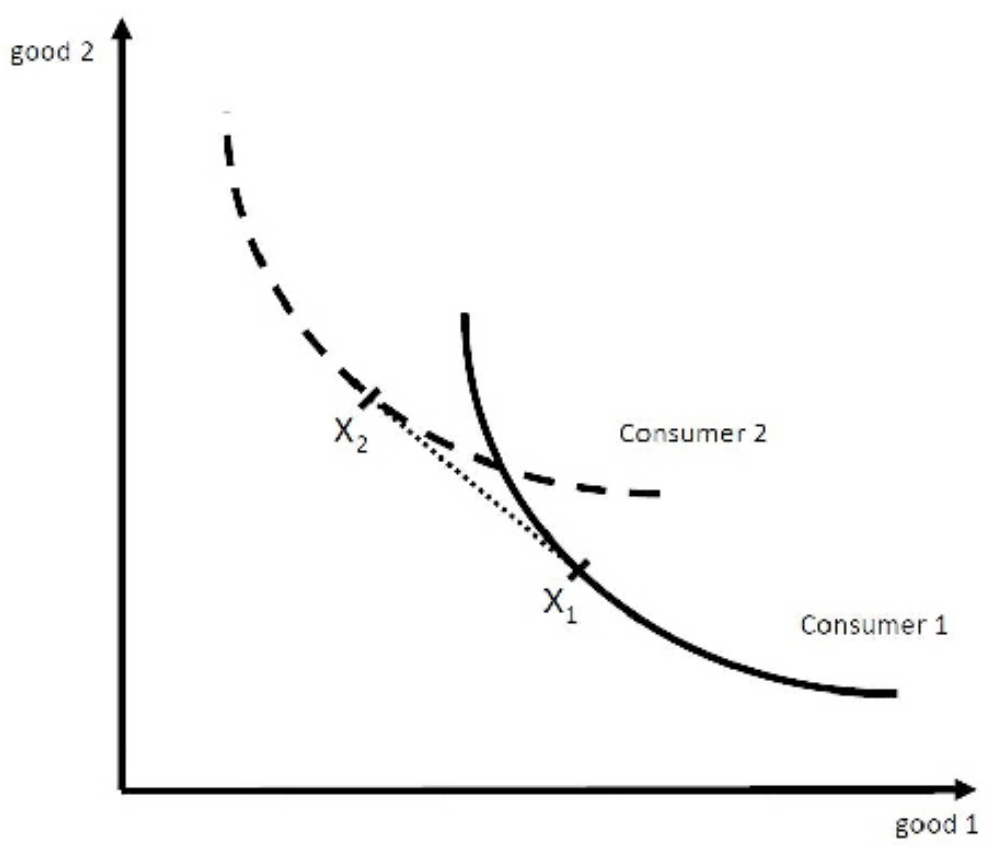

Figure 1: Kihlstrom and Mirman [3] example

"In the face of this difficulty the most natural approach, and the one taken here, is to limit comparisons to utility functions which represent the same ordinal preferences."

(Kihlstrom and Mirman, p. 366)

Now, to examine this argument more carefully, begin by noting that in the one-dimensional analysis of Arrow [1] and Pratt [5], the idea that an individual acts more risk-aversely is defined explicitly. Risk aversion is the reticence to accept an actuarially fair bet (lottery), and the comparative risk aversion of two individuals can be characterized in several ways. Of these, some pertain to observable choice behavior, rather than to properties of utility functions. One way is to compare the magnitude of the risk premium that each individual is willing to pay to avoid facing an absolute (or proportional) bet. In the particular case of a Bernoulli lottery, the magnitude of the probability premium that each individual requires to accept to face the bet also reflects comparative risk aversion.

Returning to the example, in the multivariate context, without a preexisting idea of how risk aversion is defined when there are many commodities, it is not clear what acts more risk aversely than signifies. The bundle $\frac{X_{1}+X_{2}}{2}$ is the expectation of the lottery $\widetilde{X}$, but whether the risk premium that individual 1 (or 2) is willing to pay to avoid facing the bet should be defined in terms of a positive amount of the $x$ good, or a positive amount of the $y$ good, or some (not necessarily positive) combination of the two is not apparent. In particular, the risk "premia" of 
the two individuals may have opposite signs in some directions. Alternatively, the two consumers have divergent preferences with respect to the distribution of outcomes: consumer 1 requires an increase in the probability of $X_{1}$ in order to accept the bet whereas consumer 2 requires an increase in the probability of $X_{2}$, so the direction of the probability premium is not well-defined either.

To the extent that the basis upon which one individual is said to act more risk aversely than another is not specified, it does not follow from the example in Figure 1 that risk aversion cannot be compared when ordinal preferences differ.

Although the lottery used in the example is of the simplest form (Bernoulli), it has a noteworthy feature: the two outcomes are incomparable with respect to the standard order on $\Re^{2}$. It is because of this that individuals with different preferences may disagree on their subjective rankings of the lottery outcomes. Now in fact, having argued against comparative risk aversion when preferences differ on the basis of the example in Figure 1, Kihlstrom and Mirman subsequently restrict attention to (linear) gambles whose outcomes are ordered (comparable):

"Only gambles $x+\widetilde{z} y, \widetilde{z} \geq 0$, with outcomes greater than $x$ but constrained to be in the direction $x+y$ are considered." (Kihlstrom and Mirman, p. 367)

As $\widetilde{z}$ takes on values in $\Re_{+}$only and $y$ is a positive direction, the authors thus rule out lotteries like $\widetilde{X}$ in the rest of their article.

The results they derive make more or less explicit use of this restriction. ${ }^{1}$ For example, suppose that the two goods are perfect substitutes with $u^{A}\left(x_{1}, x_{2}\right)=k\left(x_{1}+x_{2}\right), k$ an increasing and strictly concave function, and $u^{B}\left(x_{1}, x_{2}\right)=x_{1}+x_{2}$. These two utility functions represent the same ordinal preferences (the two goods are perfect substitutes), and $A$ is strictly more riskaverse than $B$ according to the authors' definition. By Proposition 1 in the paper, the risk premia should satisfy $\pi^{B}(X, Y, \widetilde{z})<\pi^{A}(X, Y, \widetilde{z})$ for any lottery $\widetilde{z}$ with support in $\Re_{+}$, originating at $X$, and in the direction $X+Y$. Without the restriction $Y \geq 0$, it is possible to take $X=(1,0)$, $Y=(-1,1)$, and $\widetilde{z}$ with support in $[0,1]$. In that case, $\pi^{A}(X, Y, \widetilde{z})=\pi^{B}(X, Y, \widetilde{z})=0$. Thus, if lotteries like $\widetilde{X}$ are considered, two indicators of comparative risk aversion that are equivalent in the one-dimensional case, the difference in risk premia and the existence of a concave utility transform, can diverge. Matters are further confounded since perfect substitutes in consumption should in principle be isomorphic to the one-dimensional (Arrow-Pratt) analysis.

There is further justification for ruling out lotteries like $\tilde{X}$ that have incomparable outcomes, if the objective is to study risk aversion with many commodities. First, if one wishes to describe attitudes towards risk, and if risk inherently involves facing better and worse outcomes, such

\footnotetext{
${ }^{1}$ In the proof of Proposition 1, the authors define $v_{x, y}^{i}(z) \equiv u^{i}(X+z Y)$ which for fixed $X, Y$ is a function of $z$ only, and for which the Arrow-Pratt risk premium can be evaluated. This requires that $Y \in \Re_{+}$, as otherwise $v_{x, y}^{i}(z)$ need not be increasing or concave.
} 


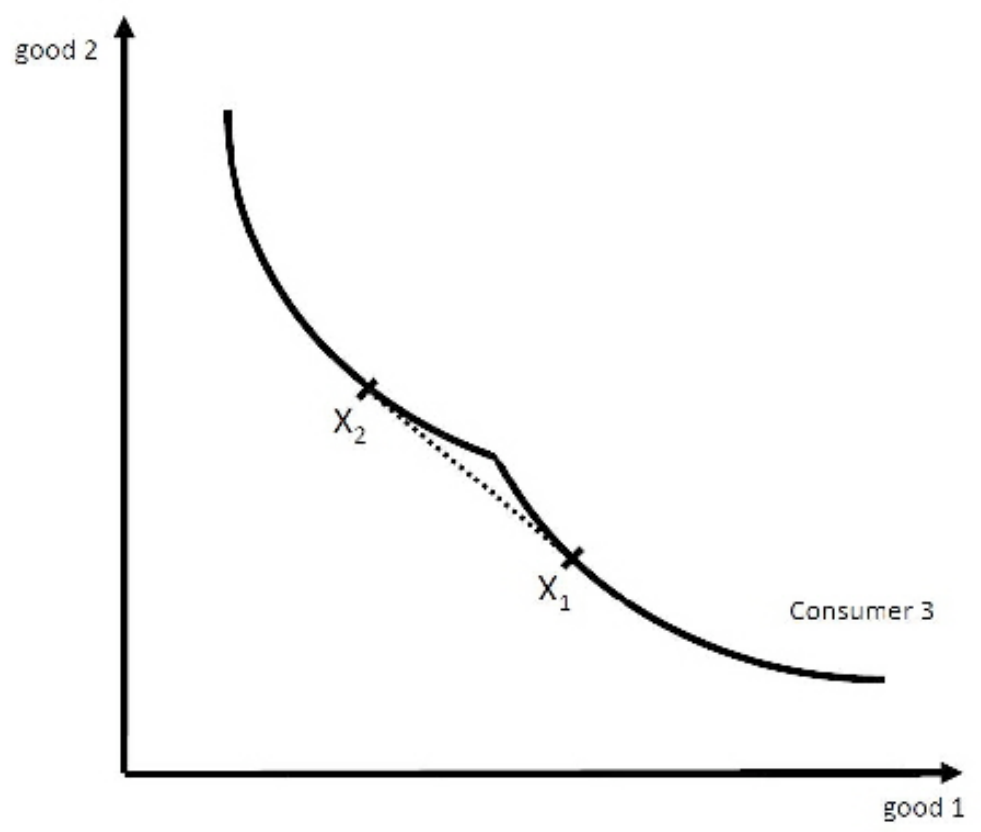

Figure 2: Nonconvexity

lotteries do not represent a risk, at least for some ordinal preferences. If different outcomes of the lottery are equally prefererred as in the perfect substitutes example, it is more correct to speak of randomness aversion rather than risk aversion. Moreover, consider the preferences in Figure 2. If preferences are not convex, and if lotteries like $\widetilde{X}$ are admissible, such configurations may arise. In the figure, regardless of the cardinal representation of utility, $E u(\widetilde{X}) \geq u(E \widetilde{X})$. If such gambles are taken to be risky and allowed in the analysis, consumer 3 may never be considered to be risk-averse, preferring the lottery to its expectation no matter what concave transform of the preferences is taken.

To summarize, it is unclear on what basis the original example in Kihlstrom and Mirman disallows comparative risk aversion when ordinal preferences differ. Moreover, this example is premised on a specific type of lottery, which is not clearly risky, and which the authors themselves rule out in their subsequent analysis. These considerations suggest that it is possible to restrict the space of lotteries as these authors do to those whose outcomes are comparable, and to also explore the possibility of comparative risk aversion for individuals with different preferences. 


\section{An Argument against Comparative Risk Aversion}

To ground the notion of acts more risk aversely than, it seems judicious to look to differences in choice behavior across individuals facing the same risky alternatives. Such an approach is developed, in a more general framework than the present one, by Bommier, Chassagnon, and Le Grand [2]. Let $R_{i}$ denote a partial order on the set of lotteries that is associated with a notion of "riskier than". These authors define comparative risk aversion in terms of choice behavior as follows:

Definition 1 (Comparative risk aversion $I$ ) $A$ is more risk averse than $B$ if for all lotteries $\widetilde{X}, \widetilde{X}^{\prime}, \widetilde{X}^{\prime} \preceq^{B} \widetilde{X}$ whenever $\widetilde{X} R_{i} \tilde{X}^{\prime}$ and $\tilde{X}^{\prime} \preceq^{A} \tilde{X}$.

With this definition, comparative risk aversion is made to hinge on the "riskier than" relation $R_{i}$, which may be specified in several ways. A natural partial order to appeal to is the meanpreserving spread (Rothschild and Stiglitz [6]). However, this partial order is uninformative for comparative risk aversion. Since no risk-averse individual ever prefers a mean-preserving spread, the application of Definition 1 yields the vacuous conclusion that all individuals are equally risk-averse.

It is then preferable to use an alternative definition of riskiness, taken from a family of relations each defined for a given $p \in(0,1)$ called $p$-spreads. The definition given here is adapted from the one Bommier et. al. advance, although they focus their attention to $p$-spreads of the utility induced by different lotteries. As ordinal preferences are allowed to differ in the present case, the only $p$-spread ordering that applies consistently over all individuals is the one that is based on the distribution functions of the random variables themselves. For simplicity finally, one may limit attention to the $p$-spreads induced by $p=0.5$, that is to the median-preserving spreads. Note that one lottery can only be a median-preserving spread of another if points in the union of the supports are comparable.

Definition 2 ( $R_{p}$, median-preserving spread) For all $\widetilde{X}, \widetilde{X}^{\prime} \in Y, \widetilde{X}$ is a median-preserving spread of $\tilde{X}^{\prime}\left(\tilde{X} R_{p} \widetilde{X}^{\prime}\right)$ if the distribution of $\tilde{X}$ crosses the distribution of $\tilde{X}^{\prime}$ once from below at some $x$ such that $F_{\widetilde{X}}(x)=F_{\widetilde{X}^{\prime}}(x)=0.5$.

If comparative riskiness of lotteries is understood in this way, then Kihlstrom and Mirman's orginal assertion is validated. It suffices, for any two individuals whose indifference curves cross at a given point, to construct a Bernoulli lottery whose high realization lies in the direction of greatest increase for one individual's utility function and whose low realization lies in the other's direction of greatest decrease. This lottery is a median (but generally not mean) preserving spread around this point, and can be constructed in such a way that it is chosen by one of the individuals and rejected by the other. 
Proposition 1 If "acts more risk-aversely" is understood with respect to Definition 1 and relation $R_{p}$ (median-preserving spread), then only individuals with the same ordinal preferences may be more risk-averse than one another.

Proof Let $u^{A}, u^{B}$ be two $\mathcal{C}^{1}$ utility functions whose indifference curves cross at a point $x$ (so $u_{1}^{A}(x) u_{2}^{B}(x) \neq u_{2}^{A}(x) u_{1}^{B}(x)$ ), and take the lottery:

$$
\widetilde{z}_{A}(\varepsilon)=\left[\left(\frac{u_{1}^{A}(x)}{\left\|\nabla u^{A}(x)\right\|} \varepsilon, \frac{u_{2}^{A}(x)}{\left\|\nabla u^{A}(x)\right\|} \varepsilon\right),\left(-\frac{u_{1}^{B}(x)}{\left\|\nabla u^{B}(x)\right\|} \varepsilon,-\frac{u_{2}^{B}(x)}{\left\|\nabla u^{B}(x)\right\|} \varepsilon\right) ; 0.5,0.5\right] .
$$

First, $x+\widetilde{z}_{A}(\varepsilon) R_{p} x$. Moreover,

$$
u^{A}\left(x+\widetilde{z}_{A}(\varepsilon)\right)=u^{A}(x)+0.5\left(\left\|\nabla u^{A}(x)\right\|-\frac{u_{1}^{A}(x) u_{1}^{B}(x)+u_{2}^{A}(x) u_{2}^{B}(x)}{\left\|\nabla u^{B}(x)\right\|}\right) \varepsilon+o\left(\varepsilon^{2}\right) .
$$

The bracketed term is strictly positive since:

$$
\begin{aligned}
& \left\|\nabla u^{A}(x)\right\|^{2}\left\|\nabla u^{B}(x)\right\|^{2}-\left(u_{1}^{A}(x) u_{1}^{B}(x)+u_{2}^{A}(x) u_{2}^{B}(x)\right)^{2} \\
= & \left(u_{1}^{A}(x) u_{2}^{B}(x)-u_{2}^{A}(x) u_{1}^{B}(x)\right)^{2}>0,
\end{aligned}
$$

so $x \preceq^{A} x+\widetilde{z}_{A}(\varepsilon)$ for $\varepsilon$ small enough. Similarly $x+\widetilde{z}_{A}(\varepsilon) \preceq^{B} x$ for $\varepsilon$ small enough. Therefore, $A$ is not more risk-averse than $B$. Analogously one can construct a lottery $\widetilde{z}_{B}(\varepsilon)$ to show that $B$ is not more risk-averse than $A$. $\square$

Several aspects of this approach warrant mention. First, taking the median-preserving spread as a notion of riskiness departs from one of the components of the orginal analysis of risk aversion, the idea of acceptance of an actuarially fair bet, since a median-preserving spread may differ in expectation from the original random variable. Second, the argument used to establish Proposition 1 involves risk attitude comparisons that are based on first-order approximations rather than the second-order terms associated with risk aversion, an approach similar in spirit to the example presented in Section 2. Finally, aside from its independent merit, the conception of risk aversion that is presented in this section thus provides a foundation for the rest of the analysis in Kihlstrom and Mirman with preferences restricted to be identical, so that risk aversion can be characterized by concave transformations.

\section{How Risk Aversion May Be Compared over Some Different Preferences}

An alternative to Definition 1 is to follow Kihlstrom and Mirman [3] in their extension of the Arrow [1] and Pratt [5] risk premium to the multivariate case. As these authors do, consider the set of lotteries to be those of the form $X+\widetilde{z} Y$, where $X, Y \in \Re_{+}^{n}$. Then comparative risk aversion may be measured by differences in directional risk premia, that is in the vector 
reduction in certain consumption that individuals are willing to accept in order to avoid facing a given gamble. This is done as follow:

Definition 3 (Multivariate risk premium) The risk premium in the direction $Y$ associated with a utility function $u^{i}$ is denoted by $\pi^{i}(X, Y, \widetilde{z})$ and defined by the equation

$$
u^{i}\left(X+\left(E \widetilde{z}-\pi^{i}\right) Y\right)=E u^{i}(X+\widetilde{z} Y) .
$$

From this measure, which may be grounded in observable choice behavior, the following definition of comparative risk aversion can be given in the spirit of the original approach. ${ }^{2}$

Definition 4 (Comparative risk aversion $I I) A$ is more risk averse than $B$ if for all $X, Y \in$ $\Re_{+}^{n}$ and all lotteries $\widetilde{z}, \pi^{B}(X, Y, \widetilde{z}) \leq \pi^{A}(X, Y, \widetilde{z})$.

The last result of this paper establishes that if "acts more risk-aversely" is understood with respect to Definition 4, then some individuals with differing ordinal preferences are more riskaverse than one another. In passing, note that trivially any concave utility function exhibits more risk aversion than $u\left(x_{1}, x_{2}\right)=x_{1}+x_{2}$, but more susbtantial examples may be devised.

Consider the pair of quasilinear utility functions:

$$
u^{A}\left(x_{1}, x_{2}\right)=x_{1}+v^{A}\left(x_{2}\right), u^{B}\left(x_{1}, x_{2}\right)=x_{1}+v^{B}\left(x_{2}\right),
$$

where $v^{A}$ and $v^{B}$ are $\mathcal{C}^{2}$ functions such that $v^{B \prime} \leq v^{A \prime}$ and $-\frac{v^{B \prime \prime}}{v^{B \prime}} \leq-\frac{v^{A \prime \prime}}{v^{A \prime}}$. Then comparisons of risk aversion are justified for these preferences. In words, if a weak single-crossing condition holds then comparative risk aversion for quasilinear preferences is determined by the one-dimensional risk aversion of the functions $v^{i}$.

Proposition $2 A$ is more risk-averse than $B$ in the sense of Definition 4.

Proof Let $v^{A}=k \circ v^{B}$ with $k^{\prime} \geq 1$ and $k$ concave. For an arbitrary risk $\widetilde{z}$ and with the quasilinear utility specification, from the definition of $\pi^{A}$ :

$$
\begin{aligned}
-\pi^{A} y_{1}+v^{A}\left(x_{2}+\left(E \widetilde{z}-\pi^{A}\right) y_{2}\right) & =E v^{A}\left(x_{2}+\widetilde{z} y_{2}\right) \\
& =E k\left(v^{B}\left(x_{2}+\widetilde{z} y_{2}\right)\right) \\
& \leq k E\left(v^{B}\left(x_{2}+\widetilde{z} y_{2}\right)\right) \\
& =k\left(-\pi^{B} y_{1}+v^{B}\left(x_{2}+\left(E \widetilde{z}-\pi^{B}\right) y_{2}\right)\right) \\
& =k\left(v^{B}\left(x_{2}+\left(E \widetilde{z}-\pi^{B}\right) y_{2}\right)\right)-\pi^{B} y_{1} k^{\prime}\left(x^{*}\right),
\end{aligned}
$$

\footnotetext{
${ }^{2}$ To be exact, Kihlstrom and Mirman begin by defining comparative risk aversion by means of concave transforms of the utility function, which they later show to be equivalent to directional risk premia:

"Thus in spite of the fact that the value of the directional risk premium varies with the direction, risk aversion comparisons of utility functions, obtained by comparing directional risk premium are independent of direction." (Kihlstrom and Mirman [3], p. 368)
} 
where $x^{*} \in\left[-\pi^{B} y_{1}+v^{B}\left(x_{2}+\left(E \widetilde{z}-\pi^{B}\right) y_{2}\right), v^{B}\left(x_{2}+\left(E \widetilde{z}-\pi^{B}\right) y_{2}\right)\right]$. By the above,

$$
\left(\pi^{A}-\pi^{B} k^{\prime}\left(x^{*}\right)\right) y_{1}=v^{A}\left(x_{2}+\left(E \widetilde{z}-\pi^{A}\right) y_{2}\right)-v^{A}\left(x_{2}+\left(E \widetilde{z}-\pi^{B}\right) y_{2}\right) .
$$

As $k^{\prime}\left(x^{*}\right) \geq 1\left(\operatorname{since} v^{B \prime} \leq v^{A \prime}\right), \pi^{A}<\pi^{B} \Rightarrow v^{A}\left(x_{2}+\left(E \widetilde{z}-\pi^{A}\right) y_{2}\right)<v^{A}\left(x_{2}+\left(E \widetilde{z}-\pi^{B}\right) y_{2}\right)$, a contradiction.

To further illustrate, with an arbitrary small mean zero risk $\widetilde{z}$, expanding the definition of the risk premium gives:

$$
\pi^{i}(X, Y, \widetilde{z}) \approx-\frac{\sigma^{2}}{2} \frac{y_{2}^{2} v^{i \prime \prime}\left(x_{2}\right)}{y_{1}+y_{2} v^{i \prime}\left(x_{2}\right)},
$$

so $\pi^{B}(X, Y, \widetilde{z}) \leq \pi^{A}(X, Y, \widetilde{z})$.

This extension of the one-dimensional Arrow-Pratt analysis is not a trivial one. Take $v^{A}\left(x_{2}\right)=-e^{-x_{2}}$ and $v^{B}\left(x_{2}\right)=-2 e^{-0.9 x_{2}}$, so $-\frac{v^{B \prime \prime}}{v^{B^{\prime}}}<-\frac{v^{A \prime \prime}}{v^{A \prime}}$ but $v^{B \prime}>v^{A \prime}$. It can be veri-

fied in the small mean zero risk case that for $x_{2} \approx 0$ and $y_{1}=y_{2}, \frac{\pi^{A}}{\pi^{B}} \approx 0.86$, that is that $A$ has a lower risk premium than $B$ for some lotteries. Thus some other restriction beyond the one-dimensional risk aversion of $v^{i}$ like the single-crossing condition given above is indeed necessary for comparative risk aversion.

To conclude, in the one-dimensional case pioneered by Arrow and Pratt as well as the rsetricted extension of Kihlstrom and Mirman, comparative risk aversion is associated with concave transformations of utility, but the above suggests that the relationship needn't be so simple in several dimensions. A more sytematic study and application of comparative risk aversion with many goods can thus be envisioned for a sequel.

\section{References}

[1] Arrow, K., 1971, Essays in the Theory of Risk-Bearing, Markham, Chicago.

[2] Bommier, A., Chassagnon, A. and F. Le Grand, 2010, "Comparative Risk Aversion: A Formal Approach with Applications to Savings Behavior", forthcoming Journal of Economic Theory.

[3] Kihlstrom, R. and L. Mirman, 1974, "Risk Aversion with Many Commodities", Journal of Economic Theory, Vol. 8, No. 3, pp. 361-388.

[4] Laffont, J.-J., 1989, The Economics of Uncertainty and Information, MIT, Cambridge.

[5] Pratt, J., 1964, "Risk Aversion in the Small and the Large", Econometrica, Vol. 32, No. 1/2, pp. 122-136.

[6] Rothschild, M. and J. Stiglitz, 1970, "Increasing Risk I: A Definition", Journal of Economic Theory, Vol. 2, No. 3, pp. 225-243. 